by a properly qualified person (not necessarily medically qualified). Secondly, the clinical interpretations of a laboratory finding should be given only by a registered medical practitioner. Lastly, a positive result should be communicated immediately to the patient's own doctor.

These three conditions emphasize that medical considerations are fundamental to pregnancy testing and to any action that may follow it. As in the case of therapeutic abortion, the demarcation between "medical" and "social" reasons for a pregnancy test is becoming increasingly blurred. But it remains the doctor's job to advise his patients on every aspect of pregnancy-its avoidance, whether it is present and the action to be taken if it is-on the basis of all the information he needs, pathological tests included.

It is in this context that the launching of a commercial home pregnancy test this week must be considered. This is claimed to be a simple, accurate, and early test which women can perform on themselves before visiting their doctor. But the fact that a woman knows she might be pregnant implies that she should have medical advice whether the result is positive or negative. She may need advice on contraception if this is not being used or has failed; a decision on posssible abortion if the right indications are present; support by the social agencies and possible adoption if an unmarried girl decides to proceed with the pregnancy. And in the relatively rare event of a high titre of human chorionic gonadotrophin being found, specialist help may be needed to eliminate the presence of a hydatidiform mole or a chorionepithelioma.

Now that simple, accurate tests for pregnancy are available these should preferably be performed in the surgery, where they should be available on request. These need not necessarily be performed by the doctor himself but by a trained member of his practice team. Unfortunately, at present family doctors have little encouragement to undertake this additional work. A clear-sighted decision by the Department of Health to reimburse the extra expense involved would help to restore pregnancy testing to its rightful context-an essential part of general medical care.

\section{Laparotomy for Fever}

Most surgeons can recall one or two patients with prolonged pyrexia of initially unknown origin in whom after a long series of unhelpful diagnostic tests a definite diagnosis is made only at laparotomy. Sometimes-for example, in the case of localized intra-abdominal abscessthe exploration allows for ready cure of the condition by a simple procedure, such as drainage. In other instances, though little or no helpful treatment can be offered, as in a case of malignant disease, at least a firm knowledge of the pathological process can allow the avoidance of further unnecessary tests, or futile and sometimes upsetting treatment.

It is always a difficult matter to decide when to undertake laparotomy on such patients, and since no individual clinician is likely to accumulate a large experience of this problem we have to rely on retrospective studies from institutions or groups of surgeons over a period of some years for factual data on such questions as the mortality and morbidity risks of laparotomy, the likely yield of useful information, the diseases most likely to be encountered, and the types of treatment most often required. From such a survey of their own and other people's experience a group of surgeons from Iowa ${ }^{1}$ have made a plea for a little more readiness to undertake exploratory laparotomy in patients presenting with continuing unexplained pyrexia, and they have suggested one or two guidelines that might prove helpful in reaching a decision to operate and in the actual conduct of the operation.

Firstly, they suggest that a period of three weeks of abnormal rise of temperature is usually required before having resort to surgery. Such a minimal time period would normally permit the elimination of most commonly occurring viral and bacterial systemic infections. While this generalization would seem to be reasonable, the timing of surgical intervention must depend to some extent on the rate of any changes in the patient's general condition. Secondly, it is helpful if in addition to the pyrexia there are any other clues to the possibility of an intra-abdominal disorder, such as anaemia, disturbance of weight or appetite, or a reproducible biochemical abnormality. Disturbance of one or other of the tests of liver function was more frequently encountered in the Iowa survey than other abnormalities, and therefore it has been suggested that a little more weight might be given to such a finding than to other factors. It is disappointing that these biochemical changes were often of a non-specific nature and did not necessarily correlate with the ultimate diagnoses. There is thus a shortage of specific signs which might act as leaders to the clinician in helping to arrive at a decision for laparotomy. Hence the difficulty of generalizing about either the need for surgical exploration or its timing. The two sides must be weighed-namely, the risk of laparotomy when no definitive surgical procedure may be required, and the risk of overlooking an easily correctable lesion which, untreated, may have serious consequences.

The few reports which have been published suggest that positive results from abdominal laparotomy are encountered in about two-thirds of the patients submitted to it, and of these about $40 \%$ prove to have malignant disease. The majority of the remainder have some form of intraabdominal infection. It might be argued that even in those who prove to have incurable malignant disease it is of value to make the diagnosis certain. It might also be argued that in those who have a completely negative laparotomy the exclusion of intra-abdominal disease may be a worthwhile contribution to the management of the patient. Much more difficult to ascertain is the number of patients who prove to have had missed remediable abdominal disorders among those who are not submitted to timely laparotomy, but who are subjected to unduly prolonged observation and blind conservative treatment. It seems likely that the number of such unfortunates is small, but if laparotomy is a relatively safe procedure in such patients then a case could certainly be made out for both wider and earlier application.

A third question raised by the Iowa surgeons is the advisability of performing splenectomy when the laparotomy is otherwise apparently negative. Certainly it would seem a reasonable procedure if there were any gross signs of abnormality either of size or consistency of this organ, but it would seem much less justified when the spleen appears normal. On balance there would seem little justification for removal of the apparently normal spleen in these circumstances. 
This is a difficult clinical problem, and it will remain largely a matter of experience to determine if and when surgical exploration of the abdomen is performed on patients presenting with continued unexplained fever. Any clue to the possible existence of an intra-abdominal lesion, even a vague one, can be extremely helpful in reaching this decision. There will still be a few patients in whom there are no obvious clues but who may still require surgical exploration. Perhaps these patients might be best served by the combination of a surgically minded physician and a conservative surgeon.

1 Ben-Shoshan, M. Gius, J. A., and Smith, I. M., Surgery, Gynecology and Obstetrics, 1971, 132, 994.

\section{Volvulus of the Stomach}

Most viscera have a single blood supply which runs in the mesentery, so that volvulus of the organ rapidly imperils the blood supply and produces gangrene. The stomach is an exception, for its blood supply is so rich and varied that volvulus usually presents as a chronic disorder. Acute gastric volvulus leading to gangrene is a rarity, and most surgeons will deal only with one or two such patients in a professional lifetime.

There are two main varieties of gastric volvulus. The first is organo-axial, which is rotation round the long axis of the stomach (the line between pylorus and cardia). The second is mesenterio-axial, which is the rotation of the stomach from right to left or left to right about the long axis of the gastro-hepatic ligament (an imaginary line drawn through the mid lesser curve and mid greater curve). In a recent review of published reports $59 \%$ of cases were found to be organo-axial and $29 \%$ mesentero-axial; the remainder were either mixed or unclassified. ${ }^{1}$ The sexes are equally affected, and the condition is commonest in the fifth decade of life.

For volvulus to occur there must be some ligamentous laxity, for the stomach is held at its oesophageal end by the ligaments round the hiatus and at its distal end by the descending part of the duodenum becoming retroperitoneal. There is usually an obvious predisposing cause, the commonest being a para-oesophageal hiatus hernia. In this condition the stomach rolls upwards into the posterior mediastinum, thus producing an organo-axial volvulus. Though the whole stomach may lie upside down in the chest, the condition may be virtually symptomless. Other diaphragmatic hernias resulting from trauma or congenital defects may occasionally be the underlying cause.

Eventration of the left diaphragm is now recognized as an important predisposing cause. ${ }^{2}$ The large space beneath the left cupola of the diaphragm is filled by the colon, which in turn pulls up the greater curve of the stomach, causing organo-axial volvulus. Other predisposing causes are pyloric or duodenal obstruction, which produces chronic dilatation of the stomach. It is suggested that a heavy meal may also predispose to the condition by approximating the pylorus and cardia.

Acute volvulus presents as an emergency with severe upper abdominal pain, which is accompanied by three important features-Borchadt's triad. They are, firstly, initial vomiting followed by retching and an inability to vomit; secondly, epigastric distension; and, thirdly, the inability to pass a nasogastric tube. Attempts to investigate the patient by barium or other contrast medium show complete obstruction at the lower end of the oesophagus. Urgent exploration is required, otherwise gangrene of the stomach is likely to necessitate resection. The mortality rate of the condition has been estimated at $30-40 \% .^{2}$

Chronic recurrent volvulus is the commoner variety, though it is still an unusual condition. It is difficult to diagnose clinically, but the patient usually gives a history of attacks of epigastric pain associated with a feeling of distension, and despite eructation there is no relief of the symptoms. The diagnosis becomes apparent on barium examination, when characteristic distortion of the stomach is seen. Many patients have only minimal symptoms and require no treatment. When the symptoms are disabling, surgical treatment should be directed towards removing the cause when this is possible. Para-oesophageal or other diaphragmatic hernias are dealt with by reduction and repair. Relief of pyloric or duodenal obstruction is needed if present. For those patients with eventration of the diaphragm various methods of treatment such as gastropexy, partial gastrectomy, anastomosis of the gastric antrum to the fundus, and gastrojejunostomy have all been tried with different degrees of success. The most logical and most successful operation is the so-called colonic displacement of the stomach. ${ }^{2}$ In this procedure the greater curve of the stomach is completely freed from the colon so that the transverse colon and great omentum can roll up under the left diaphragm without dragging the stomach after them. A gastropexy is added after this procedure. At long-term follow up 12 out of 14 patients showed a successful result after this procedure. ${ }^{2}$

1 Wastell, C., and Ellis, H., British Fournal of Surgery, 1971, 58, 557. 2 Tanner, N. C., American fournal of Surgery, 1968, 115, 505.

\section{Colleagues in Health Care}

One of the best concepts to emerge from general practice in the last few years has been that of the health team, comprising not only doctors and nurses but social workers, health visitors, medical secretaries, and practice managers. Nevertheless, descriptions of the part played by these invaluable colleagues have almost always included one comment: that at the start of an attachment scheme doctors had relatively little idea of either the qualifications for or the proper role of the particular discipline. And for their part the other members of the practice team have sometimes complained of initial misunderstandings, particularly in the early days ("the majority of doctors ... . were totally alienated from the health visitors, and . . . attitudes ranged from indifference to hostility"). ${ }^{1}$

This week (p. 479) we start a new series by a special correspondent on "Colleagues in Health Care". This will inciude descriptions of the qualifications and training of a variety of people, both in the domiciliary health team and outside it. We hope that at a time when the health services are moving towards a closer integration these will seem apposite.

1 Hockey, L., Feeling the Pulse. London, Queen's Institute of District Nursing, 1966. See B.M.7. 1966, 2, 1609. 